\title{
Trap filled limit and high current-voltage characteristics of organic diodes with non-zero Schottky barrier
}

\author{
Pankaj Kumar $^{1,4}$, S C Jain ${ }^{1,3}$, Vikram Kumar ${ }^{1}$, Suresh Chand ${ }^{1}$ and \\ R P Tandon ${ }^{2}$ \\ ${ }^{1}$ Center for Organic Electronics, National Physical Laboratory, Dr K S Krishnan road, \\ New Delhi-110 012, India \\ ${ }^{2}$ Department of Physics and Astrophysics, University of Delhi, Delhi-110007, India \\ E-mail:pankaj@mail.nplindia.ernet.in
}

Received 24 March 2008, in final form 13 May 2008

Published 17 July 2008

Online at stacks.iop.org/JPhysD/41/155108

\begin{abstract}
The analytical expressions for trap filled limit voltage $\left(V_{\mathrm{TFL}}^{\prime}\right)$ and current-voltage characteristics for non-zero organic Schottky barrier are derived theoretically. The theoretical results are validated experimentally. In this case, the injected free charge carrier density at the contact is not infinitely large but a finite number $p(0)$. For an exponential distribution of traps the maximum possible number of traps that can be filled in a sample is $H_{b}^{\prime}=H_{b}\left(\left(p(0) / N_{v}\right)\right)^{1 / l}$, where $l=T_{\mathrm{c}} / T, T_{\mathrm{c}}$ is the characteristic temperature of trap distribution. The use of Fermi-Dirac statistics causes a maximum error of only $6.9 \%$ in $H_{b}^{\prime}$. The analytical expression for $V_{\mathrm{TFL}}^{\prime}$ is shown to be $V_{\mathrm{TFL}}^{\prime}=0.5 q H_{b}^{\prime} d^{2} / \varepsilon \varepsilon_{0}$, where $d$ is the sample thickness. As the applied voltage increases and if $p(0)>H_{b}^{\prime}, V^{2}$ law is obtained over a considerable range of applied voltage. However, the curves change to Ohm's law as the voltage increases beyond this range. If $p(0)<H_{b}^{\prime}, V^{2}$ law is not obtained and the curves directly go to Ohm's law. Experimental results of ITO/PEDOT : PSS/poly(2-methoxy-5(2-ethyhexyloxy)1,4-phenylenevinylene)(MEH-PPV)/Au and ITO/PEDOT : PSS/poly(3-hexyl thiophene)(P3HT)/Au Schottky diodes are reported. The experimental results show excellent agreement with the theory.
\end{abstract}

(Some figures in this article are in colour only in the electronic version)

\section{Introduction}

Organic electronics has emerged as a revolutionary area of research due to its potential application in the next generation solar cells, displays, transistors, lasers and other devices [1-5]. Current flow through such devices determines the performance of the devices. The current-voltage $(J-V)$ characteristics of insulators (including organics) containing traps were modelled by Mark and Helfrich [6]. For the exponential distribution of traps in energy space the following $J-V$ relation, known as

\footnotetext{
3 Former address: IMEC, Kapeldreef 75, 3001 Leuven, Belgium.

${ }^{4}$ Author to whom correspondence should be addressed.
}

power law, was derived:

$$
J=q^{1-l} \mu N_{v}\left(\frac{l}{l+1} \frac{\varepsilon \varepsilon_{0}}{H_{b}}\right)^{l}\left(\frac{2 l+1}{l+1}\right)^{l+1} \frac{V^{l+1}}{d^{2 l+1}},
$$

where $q$ is the elementary charge, $\mu$ is the carrier mobility, $\varepsilon$ and $\varepsilon_{0}$ are dielectric constants of the material and permittivity of the free space, respectively, $d$ is the sample thickness, $H_{b}$ is the total trap density, $N_{v}$ is the effective density of states and $l=T_{\mathrm{c}} / T$, where $T_{\mathrm{c}}$ is the characteristic temperature of the trap distribution. This equation has been used extensively to interpret the experimental $J-V$ data in conjugated polymers and several other large band gap inorganic materials [7-10]. Equation (1) is based on 
two assumptions: (i) the injecting contact is Ohmic (i.e. the Schottky barrier is zero) and the contact is capable of injecting infinitely large concentration of charge carriers (the Schottky barrier is illustrated schematically in figure 1), (ii) the free charge carrier concentration $(p(x))$ is negligibly small compared with the trapped charge carrier density $\left(p_{\mathrm{t}}(x)\right)$. In early work (in the 1970s and 1980s) the experiments were generally performed at relatively low voltages and these approximations were valid for a good approximation. In modern technology these two approximations put severe restrictions on the usefulness of this equation.

Jain et al [11] demonstrated that at very high voltages $p(x)$ does not remain negligible compared with $p_{\mathrm{t}}(x)$. In this case, an analytical solution cannot be obtained thus equation (1) does not remain valid and the continuity and Poisson's equations are solved numerically. The numerical calculations were made on the assumption that the Schottky barrier is zero. Recently Jain et al [12] have developed an analytical model, which gives $J-V$ curves at high voltages beyond the trap filled limit (TFL). The model also yields an expression for the TFL and the voltage $\left(V_{\text {Mott }}\right)$ at which Mott's $V^{2}$ law becomes valid. The post-TFL $J-V$ curves become independent of the trap distribution in the energy space. The model is important because $J-V$ curves at high voltages can now be interpreted using this model. However, this model also uses the boundary condition that the Schottky barrier is zero. In most electrode materials and

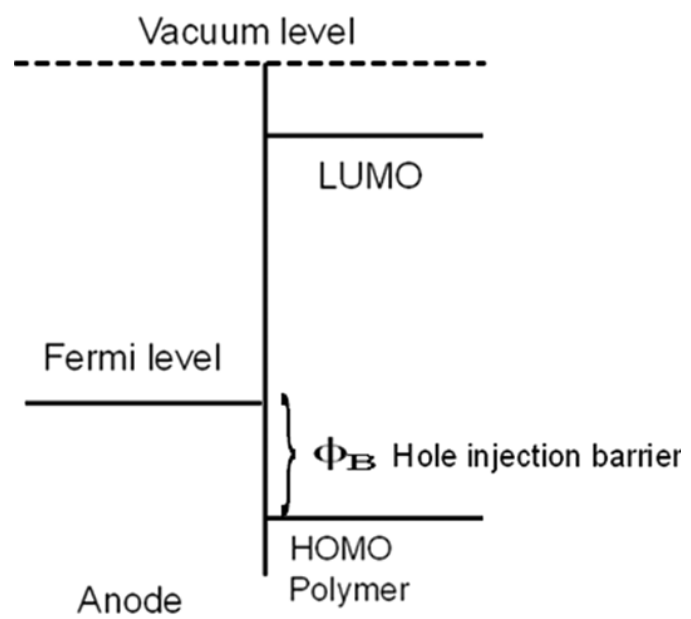

Figure 1. Typical band diagram of a polymer in contact with a metal anode. Carriers have not yet migrated across the interface to equalize the Fermi level. organics used in organic devices, the injection Schottky barrier $\left(\phi_{\mathrm{B}}\right)$ is not zero [13-17]. In a typical case of MEH-PPV and ITO the value of $\phi_{\mathrm{B}}$ is $0.4 \mathrm{eV}[18,19]$. Schottky barriers in some of the other metal-organic combinations, commonly used in the literature, are shown in table 1 . We present here the analytical model for the $V_{\mathrm{TFL}}^{\prime}$ and the $J-V$ characteristics beyond it, for a Schottky diode with a non-zero injection barrier.

\section{Theory}

\subsection{Maximum value of $p_{t}$}

We report here the solution of $J-V$ characteristics and TFL by taking into account the effect of free charge carrier density and the non-zero Schottky barriers. The traps are assumed to be exponentially distributed in the energy space. Eliminating $p(x)$ between the continuity and Poisson's equations and integrating for field $F$ over the thickness $d$ we obtain

$$
\begin{aligned}
& \int_{0}^{d} \mathrm{~d} x \\
& \quad=\frac{\varepsilon \varepsilon_{0}}{q} \int_{F(0)}^{F(d)} \frac{1}{\frac{J}{q \mu F(x)}+H_{b}\left(\frac{J}{q \mu F(x) N_{v}}\right)^{1 / l}} \mathrm{~d} F(x),
\end{aligned}
$$

where $F(0)$ and $F(d)$ are the electric fields at the injecting contact at $x=0$ and at the exit contact at $x=d$, respectively. Note that equation (2) contains a definite integral, which is equivalent to using a boundary condition that at $x=0$, the field is $F(0)$ and $p(0)$ is not infinity, as taken in the earlier paper [11]. $F(0)$ can be obtained from the equation

$$
J=q \mu p(0) F(0)
$$

Equations (2) and (3) are solved for given currents $J$. The corresponding voltages are calculated using the boundary condition

$$
V=\int_{0}^{d} F(x) \mathrm{d} x .
$$

The complete $J-V$ characteristics are obtained by solving equation (2) along with equations (3) and (4), numerically. The maximum free charge carrier density that can exist at any point inside the sample will be $p(0)$. We assume that the traps below the Fermi level are filled and the ones above it are empty. The

Table 1. Values of Schottky barriers $\phi_{\mathrm{B}}$ and injected charge carrier densities of electrons $\{n(0)\}$ and holes $\{p(0)\}$ for conducting organic PPV for different cathode and anode materials, usually used in organic semiconductor devices. Following values are at $300 \mathrm{~K}$ and the density of carriers at the Fermi level of the contacting metal is assumed to be $N_{0}=10^{19} \mathrm{~cm}^{-3}$.

\begin{tabular}{llllc}
\hline Cathode & Work function $(\mathrm{eV})$ & LUMO PPV $(\mathrm{eV})$ & Interface barrier $\left(\phi_{\mathrm{B}}\right)(\mathrm{eV})$ & $n(0)\left(\mathrm{cm}^{-3}\right)$ \\
\hline $\mathrm{Ca}$ & 2.9 & 2.7 & 0.2 & $4.38 \times 10^{15}$ \\
$\mathrm{Mg}$ & 3.7 & 2.7 & 1.0 & $1.6 \times 10^{2}$ \\
$\mathrm{Al}$ & 4.2 & 2.7 & 1.5 & $6.4 \times 10^{-7}$ \\
Anode & Work function $(\mathrm{eV})$ & HOMO PPV $(\mathrm{eV})$ & Interface barrier $\left(\phi_{\mathrm{B}}\right)(\mathrm{eV})$ & $p(0)\left(\mathrm{cm}^{-3}\right)$ \\
\hline $\mathrm{Au}$ & 5.1 & 5.3 & 0.2 & $4.38 \times 10^{15}$ \\
$\mathrm{ITO}$ & 4.8 & 5.3 & 0.5 & $4.0 \times 10^{10}$ \\
$\mathrm{Ag}$ & 4.5 & 5.3 & 0.8 & $3.67 \times 10^{5}$ \\
\hline
\end{tabular}



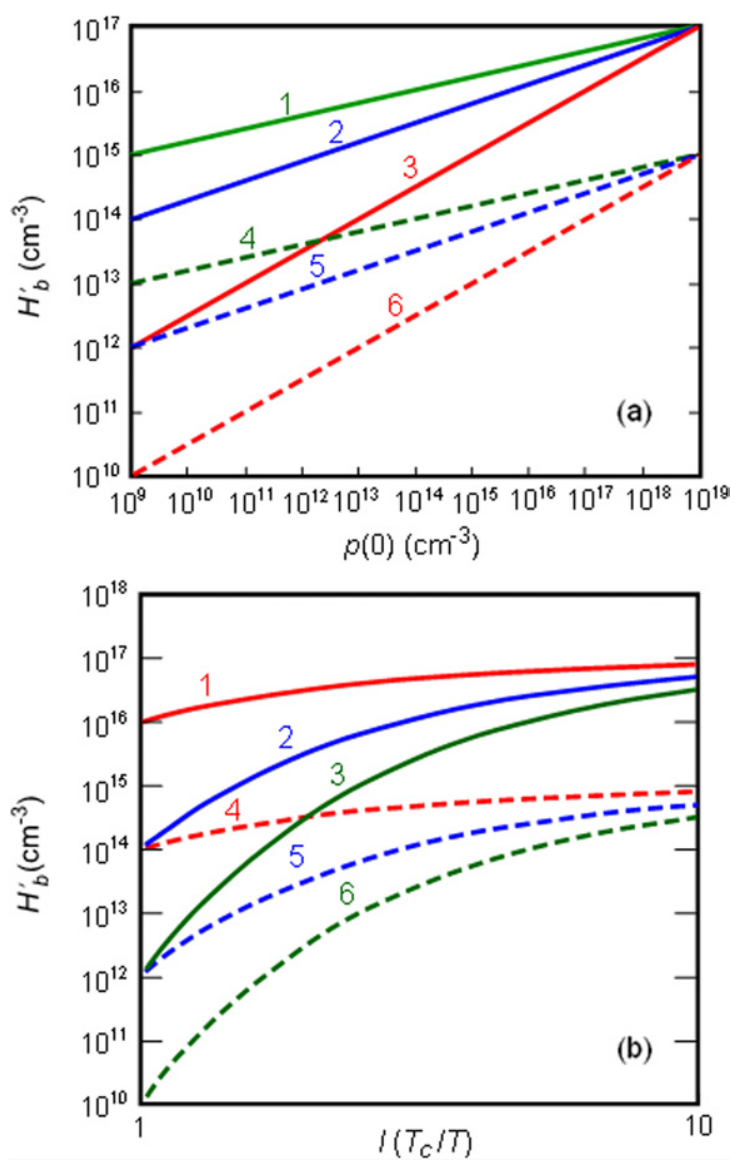

Figure 2. (a) Variation of $H_{b}^{\prime}$ with $p(0)$. The different curves are for different values of $H_{b}$ and $l$. Solid lines 1,2 and 3 are for $H_{b}=10^{17} \mathrm{~cm}^{-3}$ and $l=5,3.3$ and 2, respectively, while the dashed lines 4, 5 and 6 are for $H_{b}=10^{15}$ and $l=5,3.3$ and 2, respectively. (b) Variation of $H_{b}^{\prime}$ with $l$. Different curves are for different values of $p(0)$ and $H_{b}$. Solid lines 1,2 and 3 are for $H_{b}=10^{17} \mathrm{~cm}^{-3}$ and $p(0)=10^{18} \mathrm{~cm}^{-3}, 10^{16} \mathrm{~cm}^{-3}$ and $10^{14} \mathrm{~cm}^{-3}$, respectively, while dashed lines 4, 5 and 6 are for $H_{b}=10^{15}$ and $p(0)=10^{18} \mathrm{~cm}^{-3}$, $10^{16} \mathrm{~cm}^{-3}$ and $10^{14} \mathrm{~cm}^{-3}$, respectively. For these calculations $T_{\mathrm{c}}=3000 \mathrm{~K}$. The value of $N_{v}$ is taken to be $10^{19} \mathrm{~cm}^{-3}$ in these calculations.

Fermi level is determined by the density of free carriers, which cannot exceed $p(0)$. To obtain the maximum number of traps that can be filled at any point $x$, we determine the Fermi level corresponding to $p(x)=p(0)$ using the Boltzmann statistics. The error caused by the use of Boltzmann statistics is discussed later. Following the method of Mark and Helfrich [6], the maximum number of traps that can be filled in a sample is obtained by

$$
H_{b}^{\prime}=H_{b}\left(\frac{p(0)}{N_{v}}\right)^{1 / l} .
$$

The voltage at which $H_{b}^{\prime}$ is filled is now the TFL ( $\left.V_{\mathrm{TFL}}^{\prime}\right)$. It is seen from equation (5) that $H_{b}^{\prime}$ depends on $p(0)$ and $l$. It also depends on $N_{v}$ and is directly proportional to $H_{b}$. The variation of $H_{b}^{\prime}$ with $p(0)$ and $l$ is shown in figures $2(a)$ and $(b)$, respectively. The solid lines 1,2 and 3 in figure $2(a)$ show the results for $H_{b}=10^{17} \mathrm{~cm}^{-3}$ and for three different values of $l$, i.e. 5, 3.3 and 2, respectively. Similarly the dashed lines 4, 5 and 6 show the results for $H_{b}=10^{15} \mathrm{~cm}^{-3}$ and the same values of $l$, i.e. $5,3.3$ and 2 , respectively. Figure $2(b)$ shows the variation of $H_{b}^{\prime}$ with $l$ for two different values of $H_{b}\left(10^{17}\right.$ (solid curves) and $10^{15} \mathrm{~cm}^{-3}$ (dashed curves)) and three values of $p(0)\left(10^{18}, 10^{16}\right.$ and $\left.10^{14} \mathrm{~cm}^{-3}\right)$. As $l$ increases to higher values the curves converge to $H_{b}$. These are the interesting results in terms of the number of traps that will be filled.

\subsection{Fermi-Dirac correction}

We used Boltzmann (B.M.) statistics in deriving equation (5). It is not clear whether a substantially different value of $H_{b}^{\prime}$ will be obtained if Fermi-Dirac (F.D.) statistics is used. We now show that the error caused by the use of B.M. statistics is negligible. For this purpose we must determine the Fermi level using F.D. statistics for a free carrier concentration $p(x)$. The error in the Fermi level determined by B.M. statistics increases with $p(x)$. The maximum value of $p(x)$ for this purpose is that for which the Fermi level touches the band edge. The error will be maximum in general when $p(x)$ is sufficiently large such that the Fermi level touches the band edge. All the traps $\left(H_{b}\right)$ are filled for this value. Any further increase in $p(x)$ will cause a larger error in the use of B.M. statistics but it is of no consequence for calculating the $J-V$ characteristics as all the traps are already filled.

We assume that at any point $x$, free carrier (holes in the present case) concentration $p(x)$ is a unique function of applied voltage and it determines the Fermi level $\left(E_{\mathrm{f}}\right)$ by the following F.D. formula [20]:

$$
p=N_{v} \frac{2}{\sqrt{\pi}} \int_{0}^{\infty} \frac{\eta^{\frac{1}{2}}}{1+\exp \left(\eta+\frac{E_{\mathrm{f}}}{k T}\right)} \mathrm{d} \eta .
$$

Here $N_{v}$ is the effective density of states, $k$ is the Boltzmann constant and $T$ is the absolute temperature. $E_{\mathrm{f}}$ is measured from the valance band edge. For low carrier density and/or high temperature equation (6) reduces to

$$
p=N_{v} \exp \left(\frac{-E_{\mathrm{f}}}{k T}\right) .
$$

For an exponential distribution of traps with $0 \mathrm{~K}$ approximation, the number of traps that are filled is given by the integration of the trap distribution from $E_{\mathrm{f}}$ to $\infty[21,22]$,

$$
p_{\mathrm{t}}=H_{b} \exp \left(\frac{-E_{\mathrm{f}}}{k T_{\mathrm{c}}}\right) \text {. }
$$

Note that the upper limit of the integration used in deriving equation (8) should be the conduction band edge $\left(E_{\mathrm{c}}\right)$; however, we have used $\infty$ as the upper limit of integration. Numerical calculations show that on this account the error in $p_{\mathrm{t}}$ is quite negligible. Using $\infty$ as the upper limit it becomes possible to get the analytical solutions. The value of $p_{\mathrm{t}}$ is obtained by eliminating $E_{\mathrm{f}}[21]$ from equations (7) and (8),

$$
p_{\mathrm{t}}=H_{b}\left(\frac{p}{N_{v}}\right)^{\frac{1}{l}} \text {. }
$$

Here $l=T_{\mathrm{c}} / T$. 


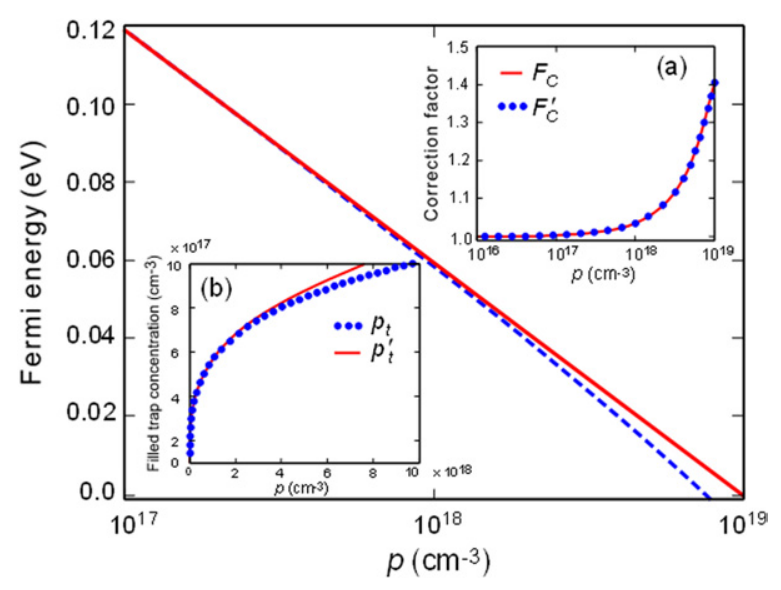

Figure 3. Fermi energies as a function of free charge carrier density $(p)$, computed from equations (6) and (7). The solid curve is for the values calculated from equation (7) and the dashed curve represents the values calculated from equation (6). Inset $(a)$ : solid curve represents the variation of the correction factor $F_{\mathrm{C}}$ with $p$ and solid circles are for the empirical relation equation (10). Inset $(b)$ : variation of $p_{\mathrm{t}}$ and $p_{\mathrm{t}}^{\prime}$ with $p$. Note that the difference in $p_{\mathrm{t}}$ and $p_{\mathrm{t}}^{\prime}$ decreases with decrease in $p$.

We calculated $E_{\mathrm{f}}$ numerically from equations (6) and (7) for different values of $p$, as shown in figure 3 . For these calculations $N_{v}$ used is $10^{19} \mathrm{~cm}^{-3}$. The values of $E_{\mathrm{f}}$ calculated from equation (6) are shown by the dashed curve while the solid curve represents the values calculated, using equation (7). It is seen that for $p>1 \times 10^{18} \mathrm{~cm}^{-3}$ the Fermi energy $\left(E_{\mathrm{f}}\right)$ from equation (6) is less than that from equation (7). At very large carrier concentration, for example $p=7.5 \times 10^{18} \mathrm{~cm}^{-3}$, the Fermi energy from equation (7) is about 11 times that from equation (6). If $E_{\mathrm{f}}$ is not several $k T$ away from the band edge equation (6) becomes necessary to determine $E_{\mathrm{f}}$ for a given concentration of free carriers.

As we change from equation (7) to equation (6) the Fermi level $E_{\mathrm{f}}$ decreases for the given $p$, i.e. the Fermi level comes closer to the band edge. Now it is obvious that the use of F.D statistics will give a larger value of $p_{\mathrm{t}}$ (see equation (8)). Therefore to get the correct value of $p_{\mathrm{t}}$ from equation (9) we will have to increase the free carrier concentration $p$. The advantage of this procedure is that we can still use the expression equation (9) provided we use the 'fictitious' value of $p$ as a mathematical artifice. Let us consider $p_{\mathrm{f}}$ as the new value of free carriers and define $F_{\mathrm{C}}$ as the correction factor equal to $p_{\mathrm{f}} / p$. The correction factor $F_{\mathrm{C}}$ can be calculated using a polynomial $F_{\mathrm{C}}^{\prime}$ of the form

$$
\begin{aligned}
F_{\mathrm{C}}^{\prime}= & {\left[1+\frac{p}{3 N_{v}}+\left(\frac{p}{3 N_{v}}\right)^{2}-\left(\frac{p}{3 N_{v}}\right)^{3}\right.} \\
& \left.+\left(\frac{p}{3 N_{v}}\right)^{4}-\left(\frac{p}{3 N_{v}}\right)^{5}\right] .
\end{aligned}
$$

$F_{\mathrm{C}}^{\prime}$ has been obtained by the numerical fitting with $F_{\mathrm{C}}$. A comparison of computed calculations for $F_{\mathrm{C}}$ and $F_{\mathrm{C}}^{\prime}$ for different values of $p$ is shown in the inset $(a)$ of figure 3 . Note that this empirical relation is suitable only when the Fermi level is in the band gap or touches the valance band. Once the Fermi level enters the valance band this relation does not remain valid. We can write the value of $p_{\mathrm{f}}$ in terms of $p$ as

$$
\begin{aligned}
p_{\mathrm{f}}= & p\left[1+\frac{p}{3 N_{v}}+\left(\frac{p}{3 N_{v}}\right)^{2}-\left(\frac{p}{3 N_{v}}\right)^{3}\right. \\
& \left.+\left(\frac{p}{3 N_{v}}\right)^{4}-\left(\frac{p}{3 N_{v}}\right)^{5}\right] .
\end{aligned}
$$

Therefore, the correct value of $p_{\mathrm{t}}$ can now be obtained by replacing $p$ with $p_{\mathrm{f}}$ in equation (9). Let $p_{\mathrm{t}}^{\prime}$ be the correct value of the filled traps. Then,

$$
p_{\mathrm{t}}^{\prime}=H_{b}\left(\frac{p_{\mathrm{f}}}{N_{v}}\right)^{\frac{1}{l}} .
$$

In this way the calculated values of the filled traps using equations (9) and (12) for different values of $p$ are shown in the inset $(b)$ of figure 3 . The values of the parameters used for these calculations are $T_{\mathrm{c}}=1200 \mathrm{~K}, T=300 \mathrm{~K}$, $H_{b}=10^{18} \mathrm{~cm}^{-3}$ and $N_{v}=10^{19} \mathrm{~cm}^{-3}$. It is concluded that the use of equation (7) introduces an error in $p$ which is maximum when the Fermi level touches the valance band and it comes out to be $\sim 30 \%$. The error decreases rapidly as the Fermi level moves away from the valance band. It is also to be noted that the error, introduced by the use of equation (7), in the calculated filled traps increases with the free carriers till all the traps are actually filled. For free carrier density $7.67 \times 10^{18} \mathrm{~cm}^{-3}$ the error in $p_{\mathrm{t}}$ comes out to be only $6.93 \%$, which decreases with the increase in $l\left(T_{\mathrm{c}} / T\right)$. For $l=20$ the error in $p_{\mathrm{t}}$ reduces to $1.35 \%$. Although the correction in $p_{\mathrm{t}}$ at room temperature is small, the accuracy in application of equation (9) improves rapidly as temperature decreases. At very high carrier concentration when the Fermi level goes into the valance band no further error in $p_{\mathrm{t}}$ will be observed as all the traps are filled and $p_{\mathrm{t}}$ has reached its saturated value $H_{b}$. Once all the traps are filled, $J-V$ characteristics will not be in error due to the use of equation (9).

\subsection{Trap filled limit voltage for finite charge carrier density}

We now discuss the case when the traps $H_{b}^{\prime}$ are already filled. In this case Poisson's equation is given by,

$$
\frac{\mathrm{d} F(x)}{\mathrm{d} x}=\frac{q}{\varepsilon \varepsilon_{0}}\left(p(x)+H_{b}^{\prime}\right) .
$$

Let us consider three dimensionless variables: distance $(w)$, field $(u)$ and potential $(v)$ as

$$
\begin{gathered}
u=\frac{H_{b}^{\prime}}{p(x)}=\frac{q H_{b}^{\prime} \mu}{J} F(x), \\
w=\frac{q^{2} H_{b}^{\prime 2} \mu}{\varepsilon \varepsilon_{0} J} x
\end{gathered}
$$

and

$$
v=\frac{q^{3} H_{b}^{\prime 3} \mu^{2}}{\varepsilon \varepsilon_{0} J^{2}} V(x) .
$$


Integrating Poisson's equation (13) and using equations (14) and (15) we get

$$
w=u-\ln (1+u)+C,
$$

where $C$ is the constant of integration. We have introduced the constant of integration $C$ to account for non-zero electric field at the junction. Using the boundary condition that at $x=0$, $w=0$ and $u=H_{b}^{\prime} / p(0), C$ comes out to be

$$
C=\ln \left(1+\frac{H_{b}^{\prime}}{p(0)}\right)-\frac{H_{b}^{\prime}}{p(0)} .
$$

By integrating equation (14) and using equation (16) we get,

$$
\begin{aligned}
v= & \frac{u^{2}}{2}-\frac{1}{2}\left[\frac{H_{b}^{\prime}}{p(0)}\right]^{2}-u+\frac{H_{b}^{\prime}}{p(0)} \\
& +\ln (1+u)-\ln \left(1+\frac{H_{b}^{\prime}}{p(0)}\right) .
\end{aligned}
$$

Let us use a subscript $a$ to represent the values of $w, u$ and $v$ at $x=d$. Now in terms of $u_{a}, w_{a}$ and $v_{a}$, equations (17) and (19) are written as

$$
w_{a}=u_{a}-\ln \left(1+u_{a}\right)+\ln \left(1+\frac{H_{b}^{\prime}}{p(0)}\right)-\frac{H_{b}^{\prime}}{p(0)}
$$

and

$$
\begin{aligned}
v_{a}= & \frac{u_{a}^{2}}{2}-\frac{1}{2}\left[\frac{H_{b}^{\prime}}{p(0)}\right]^{2}-u_{a}+\frac{H_{b}^{\prime}}{p(0)} \\
& +\ln \left(1+u_{a}\right)-\ln \left(1+\frac{H_{b}^{\prime}}{p(0)}\right) .
\end{aligned}
$$

Now the values of $J$ and $V$ can be calculated for the different values of $u_{a}$ using the following equations:

$$
J=\frac{q^{2} H_{b}^{\prime 2} \mu d}{\varepsilon \varepsilon_{0} w_{a}}
$$

and

$$
V=\frac{q H_{b}^{\prime} d^{2}}{\varepsilon \varepsilon_{0}} \frac{v_{a}}{w_{a}^{2}} .
$$

The current and voltage can be represented in the reduced units as

$$
J_{\mathrm{R}}=\frac{1}{w_{a}}
$$

and

$$
V_{\mathrm{R}}=\frac{v_{a}}{w_{a}^{2}}
$$

Equations (20) and (21) will provide the following explicit $J-V$ relation:

$$
\frac{V}{J}=\frac{d}{q H_{b}^{\prime} \mu}\left(\frac{v_{a}}{w_{a}}\right) .
$$

This result provides complete information about the $J-V$ characteristic in the whole voltage range. The relation between $J$ and $V$ at lower and higher values of voltages can be obtained for higher and lower values of $u_{a}$, respectively. The value of

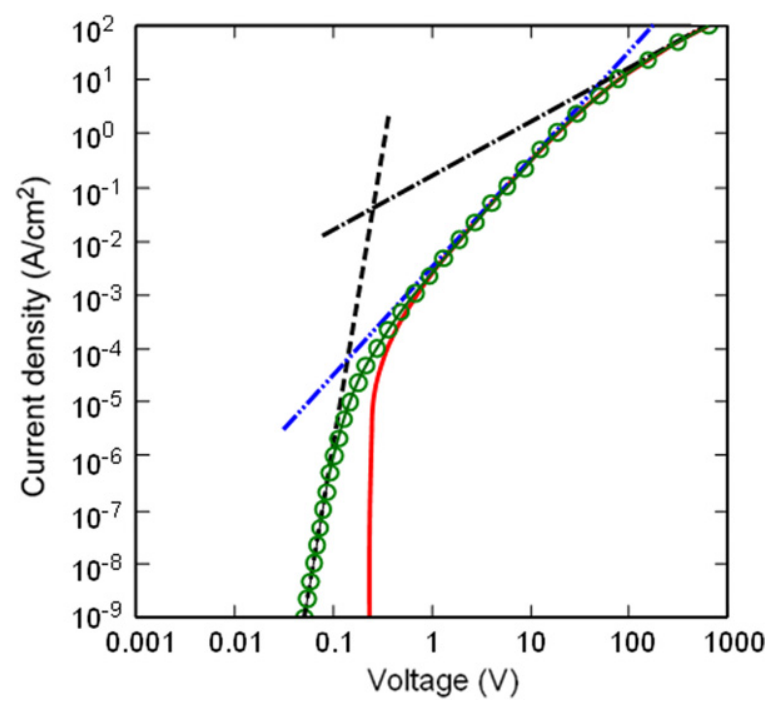

Figure 4. Computed $J-V$ characteristics of a semiconductor in contact with a metal electrode. The dashed-double dotted line represents the $V^{2}$ law and the dashed-dotted line is for Ohm's law. The dotted line is the plot of equation (1), the solid curve is a result of the new model, equation (22). The solid curve with symbols is obtained by numerical calculations without using any approximation. Note that the numerically calculated curves and plot of equation (1) will depend on $p(0)$ (see text). The values of the parameters used in the calculations are $\mu=10^{-5} \mathrm{~cm}^{2} \mathrm{~V}^{-1} \mathrm{~s}^{-1}$, $\varepsilon=3, T=300 \mathrm{~K}, T_{\mathrm{c}}=3000 \mathrm{~K}, d=100 \mathrm{~nm}, H_{b}=10^{16} \mathrm{~cm}^{-3}$, $N_{v}=10^{19} \mathrm{~cm}^{-3}$ and $p(0)=10^{18} \mathrm{~cm}^{-3}$.

$u_{a}$ is given by $H_{b}^{\prime} / p(d)$ (see equation (14)). For large values of $u_{a}, v_{a} / w_{a}^{2}$ will tend to 0.5 and $V$ will tend to

$$
V=V_{\mathrm{TFL}}^{\prime}=0.5 \frac{q H_{b}^{\prime} d^{2}}{\varepsilon \varepsilon_{0}}
$$

This is the voltage at which the maximum possible traps are filled and we call it the trap filled limit voltage $\left(V_{T F L}^{\prime}\right)$.

Figure 4 shows the plot of equation (22) (solid curve) along with the plot of equation (1) and the numerically calculated $J-V$ curve using equation (2). The dashed straight line represents the plot of equation (1) while the solid curve with circles is the numerically calculated one. The dashed-double dotted and dashed-dotted lines represent the $V^{2}$ and Ohm's laws, respectively. The values of the parameters used in the calculations are given in the caption of the figure. It is very clear from figure 4 that at low voltages the numerically calculated plot agrees well with that of equation (1). As the voltage increases the numerically calculated plot deviates from equation (1) and approaches the $V^{2}$ law. The numerically calculated follows the $V^{2}$ law for a considerable range of voltage. As the voltage increases further it again deviates from the $V^{2}$ law and approaches Ohm's law asymptotically. It is a very important result, which reveals that at higher voltages the $J-V$ curves may approach the $V^{2}$ law but for a non-zero Schottky barrier the curves will ultimately reach Ohm's law. The solid curve is practically vertical up to almost $10^{-5} \mathrm{~A} \mathrm{~cm}^{-2}$, which represents almost zero current up to $0.24 \mathrm{~V}$. The interpretation of this curve is as follows. Each applied voltage can support only a fixed amount of charge in the material. The fixed charges $H_{b}^{\prime}$ (filled traps) produce a voltage, 
which opposes the applied voltage. At $V<0.24 \mathrm{~V}$ the free carriers cannot spread and do not constitute a current. The voltage generated by the fixed charges is equal to $V_{\mathrm{TFL}}^{\prime}$. When the applied voltage exceeds $V_{\mathrm{TFL}}^{\prime}(0.24 \mathrm{~V}$, in the present case $)$ the current begins to flow and, as mentioned earlier, it follows Mott's $V^{2}$ law but within the asymptotic limit as voltage tends to infinity the curve goes to Ohm's law. It is very important to note that the occurrence of the $V^{2}$ law depends on the values of $p(0)$ and $H_{b}^{\prime}$. If $p(0)$ is less than $H_{b}^{\prime}$, the $V^{2}$ law will not be observed and the post-TFL curve will directly reach Ohm's law. These cases are discussed below in detail and analytical expressions for the $V^{2}$ law and Ohm's law have also been derived successfully.

The two cases are important, (i) $p(0)$ more than $H_{b}^{\prime}$ and (ii) $p(0)$ less than $H_{b}^{\prime}$. The minimum value of $u_{a}$ will be $H_{b}^{\prime} / p(0)$. Let us first consider the case when $p(0)$ is more than $H_{b}^{\prime}$ then $H_{b}^{\prime} / p(0)$ will be less than 1 . As voltage increases $p(d)$ increases and at a sufficiently high voltage $H_{b}^{\prime} / p(d)$ becomes much less than 1 . In other terms $u_{a}\left(H_{b}^{\prime} / p(d)\right)$ will tend to be much less than 1 . In this case the expansion of equation (17') will give

$$
w_{a}=\frac{1}{2}\left[u_{a}^{2}-\left(\frac{H_{b}^{\prime}}{p(0)}\right)^{2}\right] .
$$

Similarly the expansion of equation $\left(19^{\prime}\right)$ will give

$$
v_{a}=\frac{1}{3}\left[u_{a}^{3}-\left(\frac{H_{b}^{\prime}}{p(0)}\right)^{3}\right] .
$$

Now putting the values of $w_{a}$ and $v_{a}$ from equations (24) and (25) in equation (22), we get

$$
J=\frac{3}{2} q \mu H_{b}^{\prime}\left[\frac{u_{a}+\frac{H_{b}^{\prime}}{p(0)}}{u_{a}^{2}+\left(\frac{H_{b}^{\prime}}{p(0)}\right)^{2}+u_{a}\left(\frac{H_{b}^{\prime}}{p(0)}\right)}\right] \frac{V}{d} .
$$

Now for large values of $V, u_{a}$ will tend to $H_{b}^{\prime} / p(0)$, which is less than 1 . As $u_{a}$ tends to $H_{b}^{\prime} / p(0)$, let us consider the case when $u_{a}$ is less than 1 but much more than $H_{b}^{\prime} / p(0)$. In this case equation (26) will give

$$
J=\frac{3}{2} q \mu H_{b}^{\prime} \frac{V}{d}\left(\frac{1}{u_{a}}\right) .
$$

When the values of $u_{a}$ are less than 1 , equation (17') will give $u_{a}=\left(2 w_{a}\right)^{1 / 2}$ and ultimately equation (27) will give

$$
J=\frac{9}{8} \mu \varepsilon \varepsilon_{0} \frac{V^{2}}{d^{3}},
$$

which is the well-known Mott's $V^{2}$ law.

Now when $u_{a}$ becomes equal to $H_{b}^{\prime} / p(0)$, equation (26) gives

$$
J=q p(0) \mu \frac{V}{d} .
$$

This is the well-known Ohm's law. Equations (28) and (29) reveal that after the traps are filled, the current will first

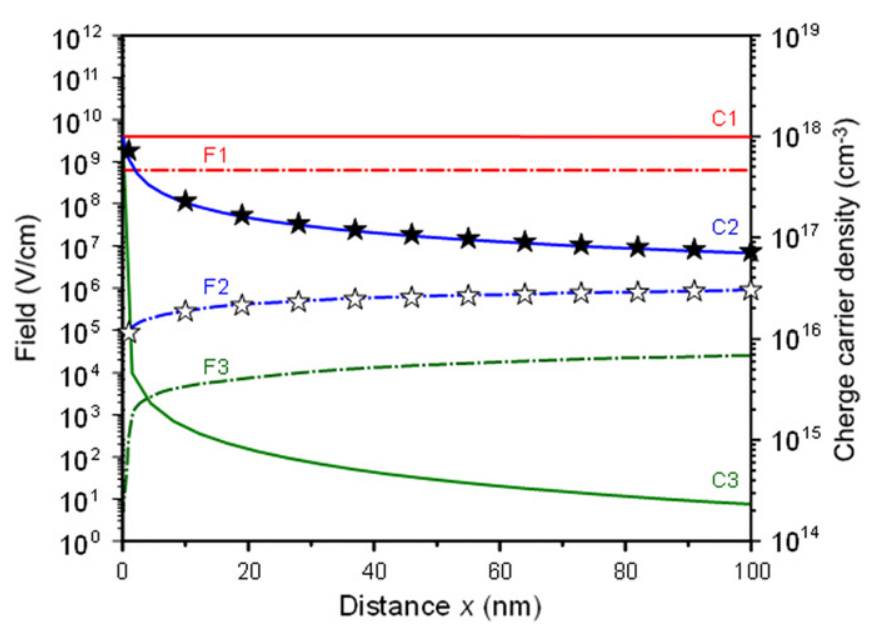

Figure 5. Calculated electric field profiles (dashed-dotted curves) and charge carrier profiles (solid curves) at different currents. The different currents used in the calculations are $J=10^{3} \mathrm{~A} \mathrm{~cm}^{-2}$ (curves $\mathrm{F} 1$ and $\mathrm{C} 1$ ), $10^{-1} \mathrm{~A} \mathrm{~cm}^{-2}$ (curves $\mathrm{F} 2$ and $\mathrm{C} 2$ ) and $10^{-5} \mathrm{~A} \mathrm{~cm}^{-2}$ (curves F3 and C3). The solid symbols represent the variation of carriers profile as $1 / \sqrt{x}$, and the open symbols represent the variation of field profile as $\sqrt{x}$, which corresponds to the $V^{2}$ law. The values of the parameters used in these calculations are the same as given in figure 4 .

go to Mott's $V^{2}$ law and then ultimately it reaches Ohm's law at high voltages. This statement is also quite clear from figure 5 , which shows the electric field and the charge carrier profiles for different currents. Filled and open symbols are for the profiles of charge carriers and electric field, respectively, when the current follows the $V^{2}$ law, given by equation (28). Note that when the current follows the $V^{2}$ law, the charge carrier density varies as $1 / \sqrt{x}$, while the electric field varies as $\sqrt{x}$. The values of the parameters used in these calculations are the same as given in the caption of figure 4 . The solid curves represent the charge carrier profiles $(\mathrm{C} 1, \mathrm{C} 2, \mathrm{C} 3)$ while the dashed-dotted curves are for the electric field profiles (F1, F2, F3). The profiles $\mathrm{C} 3$ and $\mathrm{F} 3$ are for $10^{-5} \mathrm{~A} \mathrm{~cm}^{-2}$. When the current increases further, let us take an example of $10^{-1} \mathrm{~A} \mathrm{~cm}^{-2}$, the charge carrier (C2) and the field (F2) profiles agree perfectly with the filled and open symbols, respectively. This is clear evidence that at $10^{-1} \mathrm{~A} \mathrm{~cm}^{-2}$, the current follows the $V^{2}$ law. With a further increase in the current, for example $10^{3} \mathrm{~A} \mathrm{~cm}^{-2}$, the charge carrier $(\mathrm{C} 1)$ and the electric field (F1) profiles become perfectly flat, which shows the Ohmic conduction in the sample. Therefore ultimately at high voltages the current follows Ohm's law.

Now let us consider the case when $p(0)$ is less than $H_{b}^{\prime}$. We can write $u_{a}$ in terms of $H_{b}^{\prime} / p(0)$ as $u_{a}=\left(H_{b}^{\prime} / p(0)\right)+\delta u_{a}$. As $u_{a} \rightarrow H_{b}^{\prime} / p(0), \delta u_{a} \rightarrow 0$. Considering this concept as $\delta u_{a} \rightarrow 0, w_{a}$ and $v_{a}$ will be given by

$$
w_{a}=\delta u_{a}\left(\frac{H_{b}^{\prime}}{H_{b}^{\prime}+p(0)}\right)
$$

and

$$
v_{a}=\delta u_{a}\left(\frac{H_{b}^{\prime 2}}{p(0)\left(H_{b}^{\prime}+p(0)\right)}\right) .
$$




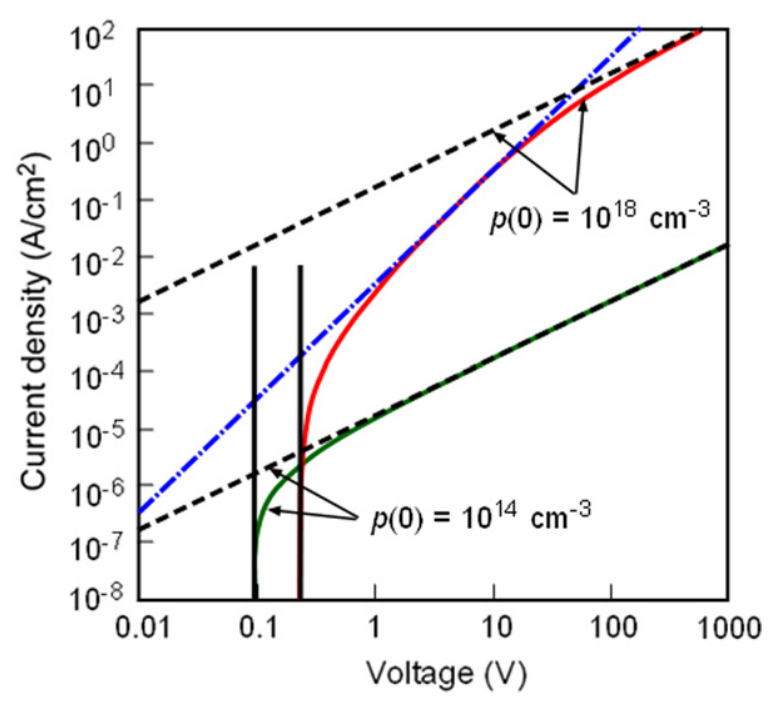

Figure 6. Post-TFL $J-V$ characteristics for two different values of $p(0)$. The dashed-dotted line represents the $V^{2}$ law and the dashed lines are for Ohm's law. The dark solid vertical lines are the values of $V_{\mathrm{TFL}}^{\prime}$, calculated using equation (23) for the two respective values of $p(0)$. The parameters used in these calculations are the same as given in figure 4.

Putting the value of $w_{a}$ and $v_{a}$ from equations (30) and (31) in equation (22) we get

$$
J=q p(0) \mu \frac{V}{d} .
$$

This is Ohm's law. Therefore for higher values of applied voltages the current tends to Ohm's law.

Figure 6 shows the plots of equation (22) for two cases, (i) when $p(0)$ is more than $H_{b}^{\prime}$ and (ii) when $p(0)$ is less than $H_{b}^{\prime}$. These calculations have been made for $H_{b}=10^{16} \mathrm{~cm}^{-3}$, and for two values of $p(0)$, i.e. $p(0)=10^{18} \mathrm{~cm}^{-3}$ and $p(0)=10^{14} \mathrm{~cm}^{-3}$. For these two cases the values of $H_{b}^{\prime}$ are $7.94 \times 10^{15} \mathrm{~cm}^{-3}$ and $3.16 \times 10^{15} \mathrm{~cm}^{-3}$, respectively. The dashed-dotted line represents the $V^{2}$ law and the dashed lines represent Ohm's law for the two values of $p(0)$. The solid vertical lines are the plots of equation (23) and represent the $V_{\mathrm{TFL}}^{\prime}$ for the two cases. It is clear that when $p(0)>H_{b}^{\prime}$, the current first follows the $V^{2}$ law and then goes to Ohm's law asymptotically. When $p(0)<H_{b}^{\prime}$, no $V^{2}$ dependence is observed and the current goes directly to Ohm's law asymptotically.

The $J-V$ characteristics and $V_{\mathrm{TFL}}^{\prime}$ calculated in figure 4 are for a specific case. All calculations will have to be made every time a different sample is considered. To avoid the repetition of calculations, we calculate universal $J-V$ characteristics for a set of given values of $p(0)$ and $H_{b}^{\prime}$, valid for all semiconductors. The universal TFL voltage and post-TFL characteristic in reduced units are obtained from equations $\left(20^{\prime}\right)$ and $\left(21^{\prime}\right)$. The universal reduced TFL voltage comes out to be 0.5 , which is independent of $p(0)$ and $H_{b}^{\prime}$. To obtain the actual $V_{\mathrm{TFL}}^{\prime}$ the universal reduced TFL voltage must be multiplied by $q H_{b}^{\prime} d^{2} / \varepsilon \varepsilon_{0}$. Ohm's law and Mott's $V^{2}$ law can also be represented in the reduced units. When $p(0)$ is less than $H_{b}^{\prime}$,

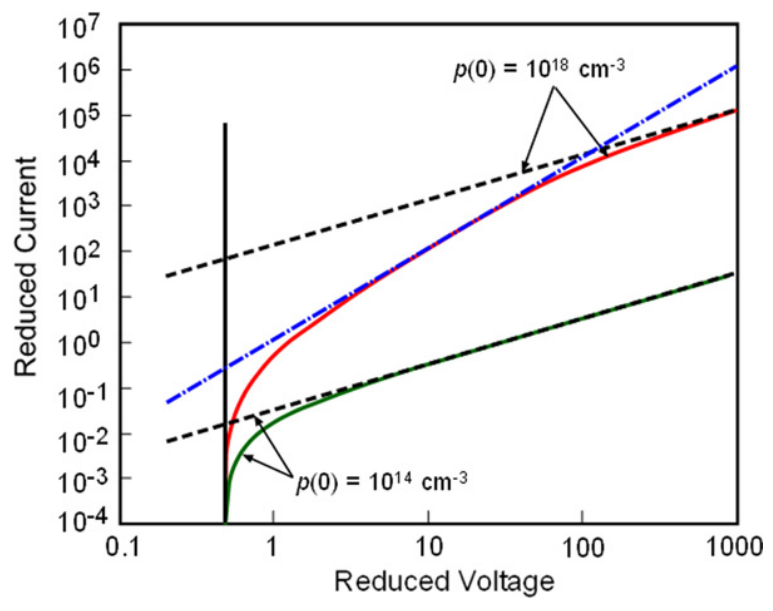

Figure 7. Computed $J-V$ characteristics of a semiconductor in reduced units for different values of $p(0)$. The dashed-dotted line represents the reduced $V^{2}$ law and the dashed lines are the reduced Ohm's law for respective values of $p(0)$. The solid dark vertical line is the universal reduced TFL. The values of the parameters used in the calculations are the same as given in figure 4

for Ohm's law, $w_{a}$ and $v_{a}$ will be given by

$$
w_{a}=\left(\frac{u_{a} p(0)-H_{b}^{\prime}}{H_{b}^{\prime}+p(0)}\right) \frac{H_{b}^{\prime}}{p(0)}
$$

and

$$
v_{a}=\left(\frac{u_{a} p(0)-H_{b}^{\prime}}{H_{b}^{\prime}+p(0)}\right) \frac{H_{b}^{\prime 2}}{p(0)^{2}} .
$$

Putting these values of $w_{a}$ and $v_{a}$ in equations $\left(20^{\prime}\right)$ and $\left(21^{\prime}\right)$, the solution gives

$$
J_{\mathrm{R}}=\frac{p(0)}{H_{b}^{\prime}} V_{\mathrm{R}},
$$

which is Ohm's law in reduced units. A similar relation can also be obtained using equations (24) and (25), when $p(0)$ is more than $H_{b}^{\prime}$. For the $V^{2}$ law in reduced units, $w_{a}$ and $v_{a}$ will be given by

$$
w_{a}=\frac{1}{2} u_{a}^{2}
$$

and

$$
v_{a}=\frac{1}{3} u_{a}^{3} .
$$

Now putting these values of $u_{a}$ and $w_{a}$ in equations $\left(20^{\prime}\right)$ and $\left(21^{\prime}\right)$, the solution gives

$$
J_{\mathrm{R}}=\frac{9}{8} V_{\mathrm{R}}^{2}
$$

which is the universal $V^{2}$ law in reduced units.

Reduced current-voltage $\left(J_{R}-V_{R}\right)$ characteristics for given values of $H_{b}$ and $p(0)$ (i.e. $H_{b}=10^{16} \mathrm{~cm}^{-3}$ and $p(0)=10^{18}$ and $10^{14} \mathrm{~cm}^{-3}$ ) are plotted in figure 7 . The dashed-dotted line represents the $V^{2}$ law while the dashed lines are for Ohm's law for respective values of $p(0)$, in reduced units. The solid vertical line represents the TFL voltage in reduced units. Figure 7 also shows that when $p(0)>H_{b}^{\prime}$, the current first follows the $V^{2}$ law and then Ohm's law. But when $p(0)<H_{b}^{\prime}$, no $V^{2}$ dependence is observed and the 
current goes directly to Ohm's law. To validate the theory we present a comparison of the theory with experimental data and TFLs have been calculated for two different conjugated polymer samples.

\section{Experimental details}

To validate the theory, the $J-V$ characteristics of the Schottky diodes based on two different conjugated polymers MEHPPV and P3HT with non-zero injection barriers are studied and reported here. Diodes were fabricated in hole only ITO/PEDOT : PSS/polymer/Au structure. The pre-patterned ITO coated glass substrates (resistivity $\sim 18 \Omega / \square$ ) were cleaned via a standard cleaning process and exposed to oxygen plasma for $5 \mathrm{~min}$. PEDOT : PSS (Aldrich) was spin coated at around $1000 \mathrm{rpm}$ on the ITO substrates and cured at $100^{\circ} \mathrm{C}$ for $1 \mathrm{~h}$ in vacuum. MEH-PPV and P3HT (Aldrich) were dissolved in ortho-dichlorobenzene and sonicated for $1 \mathrm{~h}$ at $50{ }^{\circ} \mathrm{C}$. The polymer content of the solutions was $15 \mathrm{mg} \mathrm{ml}^{-1}$. These solutions were spin coated over the PEDOT:PSS coated ITO substrates and cured at $120^{\circ} \mathrm{C}$ in a glove box in inert atmosphere for $10 \mathrm{~min}$. The final thicknesses of the P3HT and MEH-PPV layers (as measured from capacitive calculations) were $\sim 99 \mathrm{~nm}$ and $80 \mathrm{~nm}$, respectively. Finally thick gold electrodes $(500 \mathrm{~nm})$ were thermally deposited in vacuum at $4 \times 10^{-6}$ Torr, over the polymer layers through shadow masks that defined the area of the diodes to be $0.08 \mathrm{~cm}^{2}$ for both the diodes. The $J-V$ characteristics of the devices were measured using a Keithley 2400 Source measure unit, interfaced with a computer.

\section{Results and discussion}

We have discussed the temperature effects on the $J-V$ characteristics of MEH-PPV earlier in [7]. In this paper we concentrate on the interpretation of high voltage $J-V$ data including the determination of TFL and post-TFL $J-V$ curves at room temperature. The experimental $J-V$ characteristics of the MEH-PPV and P3HT diodes have been shown by symbols in figure 8. The dashed line is for Ohm's law (equation (32)). Note that at higher voltages the experimental data have a slope of less than 2 and approaches Ohm's law. No $V^{2}$ dependence of the current has been observed in the experimental data for both the diodes. Therefore from the absence of $V^{2}$ dependence and Ohm's law approach of the experimental data, it can be inferred that the diodes have non-zero injection Schottky barriers and the total trap density $\left(H_{b}\right)$ is more than $p(0)$. A comparison of the MEH-PPV and P3HT diodes data with the present theory is shown in figures $8(a)$ and $(b)$, respectively. The solid curve represents the numerically calculated $J-V$ characteristic using equation (2), the dashed-double dotted curve is the plot of equation (22) and the dashed-dotted line is the plot of equation (1). At low voltages both the numerically calculated curve and the plot of equation (1) overlap. As voltage increases to higher values the calculated curve deviates from the plot of equation (1) and bends down. Within the limit of high voltages the curve approaches Ohm's law. At low voltages experimental data show Ohmic behaviour, which can
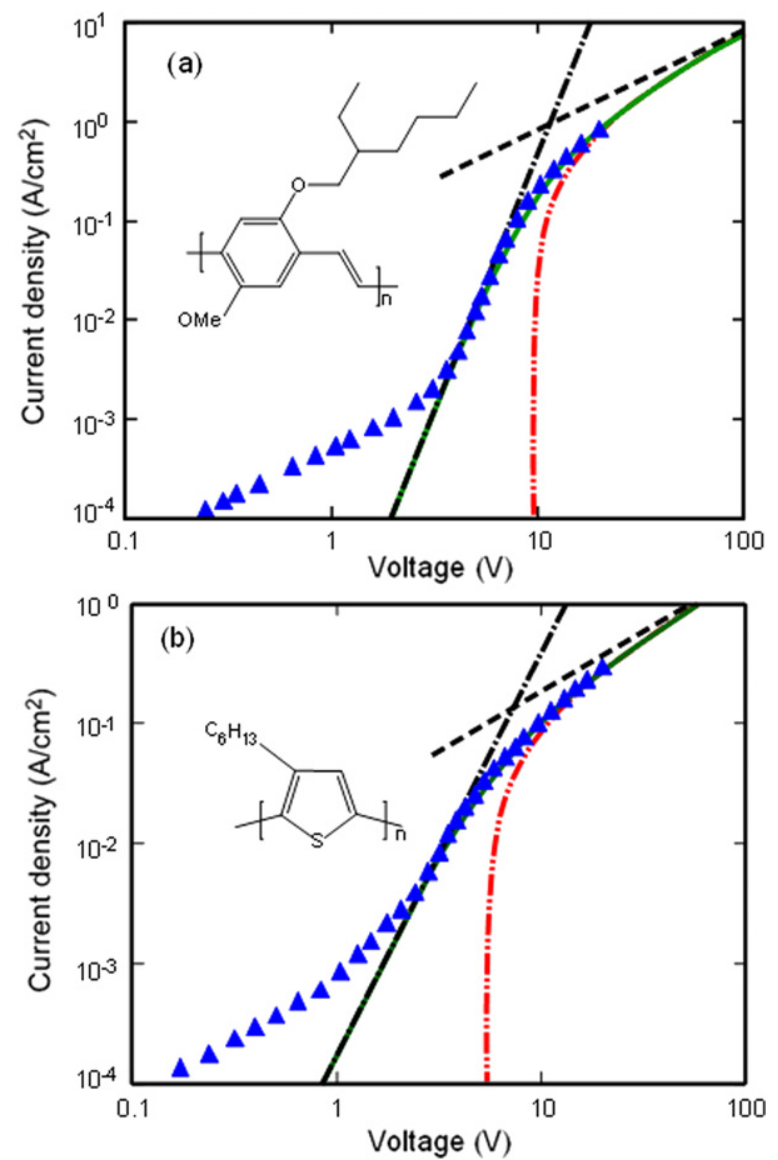

Figure 8. (a) Comparison of experimental $J-V$ characteristic of ITO/PEDOT : PSS/MEH-PPV/Au diode, with the new model. The symbols represent the experimental data at room temperature. The values of the parameters are $\mu=4.5 \times 10^{-5} \mathrm{~cm}^{2} \mathrm{~V}^{-1} \mathrm{~s}^{-1}, \varepsilon=3$, $T_{\mathrm{c}}=1250 \mathrm{~K}, d=80 \mathrm{~nm}, H_{b}=1.55 \times 10^{18} \mathrm{~cm}^{-3}, N_{v}=10^{19} \mathrm{~cm}^{-3}$, $N_{0}=10^{19} \mathrm{~cm}^{-3}$ and $p(0)=8.913 \times 10^{16} \mathrm{~cm}^{-3}$, which corresponds to $\phi=0.12 \mathrm{eV}$. The inset shows the molecular structure of MEH-PPV. (b) Comparison of experimental $J-V$ characteristic of ITO/PEDOT: PSS/P3HT/Au diode, with the new model. The symbols represent the experimental data at room temperature. The values of the parameters are $\mu=2.2 \times 10^{-4} \mathrm{~cm}^{2} \mathrm{~V}^{-1} \mathrm{~s}^{-1}, \varepsilon=3$, $T_{\mathrm{c}}=700 \mathrm{~K}, d=99 \mathrm{~nm}, H_{b}=4.7 \times 10^{18} \mathrm{~cm}^{-3}, N_{v}=10^{19} \mathrm{~cm}^{-3}$, $N_{0}=10^{19} \mathrm{~cm}^{-3}, p(0)=5.248 \times 10^{15} \mathrm{~cm}^{-3}$, which corresponds to $\phi=0.195 \mathrm{eV}$. The inset shows the molecular structure of P3HT.

be attributed to the background doping. The experimental data show very good agreement with the numerically calculated $J-$ $V$ curve in the whole voltage range. Assuming the effective density of states $\left(N_{v}\right)$ to be $10^{19} \mathrm{~cm}^{-3}$, the total trap density and characteristic temperature have been calculated to be $H_{b}=1.55 \times 10^{18} \mathrm{~cm}^{-3}$ and $T_{\mathrm{c}}=1250 \mathrm{~K}$. The values of the parameters are given in the caption of figure 8. Ohm's law, to which the experimental data approach, corresponds to $p(0)=8.913 \times 10^{16} \mathrm{~cm}^{-3}$. The injection Schottky barrier corresponding to the above value of $p(0)$ has been calculated to be $0.12 \mathrm{eV}$, which is in good agreement with the difference of work function of PEDOT : PSS and HOMO of MEH-PPV $[18,19]$. Similarly the injection Schottky barrier corresponding to the $p(0)$ for the P3HT diode has been calculated to be $0.195 \mathrm{eV}$, which is in good agreement with the difference of the work function of PEDOT: PSS and HOMO of P3HT 
[19]. The values of $V_{\mathrm{TFL}}^{\prime}$ for the two samples of MEH-PPV and P3HT have been calculated to be $9.63 \mathrm{~V}$ and $5.45 \mathrm{~V}$, respectively. Beyond the $V_{\mathrm{TFL}}^{\prime}$ experimental data agree well with equation (22). The value of $N_{v}$ used is 1-2 orders of magnitude higher [23] than the value we used here for P3HT. The $J-V$ relation remains unchanged if $N_{v}=10^{20} \mathrm{~cm}^{-3}$ is considered provided that the fitting parameter $H_{b}$ changes from $4.7 \times 10^{18}$ to $1.3 \times 10^{19} \mathrm{~cm}^{-3}$. At the present state of the art of the transport in organic materials, the values of material parameters are not uniquely determined. Therefore the values of the parameters used by us are reasonable. Jain et al [11] studied the $J-V$ characteristics of an ITO/MEHOPV5/Al Schottky diode up to $10 \mathrm{~V}$. The values of $H_{b}^{\prime}$ and $p(0)$ in this sample are $6 \times 10^{16}$ and $\sim 10^{18} \mathrm{~cm}^{-3}$, respectively. According to our theory for this sample it is expected that first the current will follow the $V^{2}$ law for a considerable range of applied voltage and finally at very high voltages it will follow Ohm's law. The experiments have been performed only up to $10 \mathrm{~V}$. As expected, the experimental data follow the $V^{2}$ law from 3 to $10 \mathrm{~V}$. Experiments have not been performed at very high voltages, therefore Ohm's law was not observed. Our theory suggests that if the experiments were performed at higher voltages for this sample, Ohm's law would have been observed.

\section{Conclusion}

Recent experiments indicate that the approximation of zero Schottky barriers in conducting organic diodes is not valid in several cases. Furthermore, measurements have been made at high voltages where no analytical theory for currents is available at present. We have developed an analytical theory, which gives $V_{\mathrm{TFL}}^{\prime}$ and the $J-V$ characteristics beyond the $V_{\mathrm{TFL}}^{\prime}$. The theory shows that if injected carrier density $p(0)$ is less than the effective trap density $H_{b}^{\prime}$, beyond TFL Ohm's law is obtained at high voltages. On the other hand, if $p(0)$ is more than $H_{b}^{\prime}$, first Mott's $V^{2}$ law is obtained at high voltages but ultimately current follows Ohm's law at higher voltages. Thus if $p(0)$ is not infinitely large, Ohm's law is always obtained in the limit of very high voltages. The carrier profiles have been calculated at different currents. When Ohm's law is applicable the carrier profile becomes constant $(p(0))$ with $x$. On the other hand, when Mott's $V^{2}$ law is applicable the carrier profile varies as $1 / \sqrt{x}$. Our experiments for MEH-PPV and P3HT show very good agreement with the theory for $p(0)$ less than $H_{b}^{\prime}$.

\section{Acknowledgments}

The authors would like to thank Dr A K Gupta and Dr S S Bawa (all from NPL, India) for their kind help and co-operation. The financial support from CSIR, India is gratefully acknowledged.

\section{References}

[1] Jain S C, Aernout T, Kapoor A K, Kumar V, Geens W, Poortmans J and Mertens R 2005 Synth. Met. 148245

[2] Gunes S, Neugebauer H and Sariciftci N S 2007 Chem. Rev. 1071324

[3] Forrest S R 2004 Nature 428911

Burroughes J H, Bradley D D C and Brown A R 1990 Nature 347539

[4] Sirringhaus H 2005 Adv. Funct. Mater. 172411

[5] Singh S, Kanetkar V R, Sridhar G, Muthuswamy V and Raja K 2003 J. Lumin. 101285

[6] Helfrich W and Mark P 1962 Z. Phys. 166370

Helfrich W and Mark P 1962 Z. Phys. 168495

[7] Kumar P, Misra A, Kamalasanan M N, Jain S C and Kumar V 2007 J. Phys. D: Appl. Phys. 40561

[8] Campbell A J, Bradly D D C and Lidzey D G 1997 J. Appl. Phys. 826326

Campbell A J, Weaver M S, Lidzey D G and Bradly D D C 1998 J. Appl. Phys. 846737

[9] Gloud R D and Rahman M S 1981 J. Phys. D: Appl. Phys. 1479

[10] Liang T, Guo X, Guan B, Guo J, Gu X, Lin Q, Wu D, Gao G, Zhu Y and Shen G 2007 Appl. Phys. Lett. 90102107

[11] Jain S C, Kapoor A K, Geens W, Pootmans J, Mertens R and Willander M 2002 J. Appl. Phys. 923752

[12] Jain A, Kumar P, Jain S C, Kumar V, Kaur R and Mehra R M 2007 J. Appl. Phys. 10094505

[13] Heil H, Steiger J, Gastel M, Ortner H, Seggem H V and Stossel M $2001 \mathrm{~J}$. Appl. Phys. 89420

[14] Parker I D 1994 J. Appl. Phys. 751656

[15] Poplavskyy D, Nelson J and Bradley D D C 2003 Appl. Phys. Lett. 83707

[16] Baldo M A and Forrest S R 2001 Phys. Rev. B 6485201

[17] Wolf U, Arkhipov V I and Bassler H 1999 Phys. Rev. B 597507

[18] Jin S H, Jang M S, Suh H S, Cho H N, Lee J H and Gal Y S 2002 Chem. Mater. 14643

[19] Chiguvare Z and Dyakonov V 2004 Phys. Rev. B 70235207

[20] Sze S M 1981 Physics of Semiconductor Devices (New York: Wiley)

[21] Kao K C and Hwang W 1981 Electrical Transport in Solids (Oxford: Pergamon)

[22] Jain S C, Willander M and Kumar V 2007 Conducting Organic Materials and Devices $\mathbf{8 1}$ (New York: Academic)

[23] Coehoorn R, Pasveer W F, Bobbert P A and Michels M A J 2005 Phys. Rev. B 72155206 